\title{
Do There Exist Innovative Perspectives in Tissue Engineering-Based Ligament Reconstruction?
}

\begin{abstract}
Ligament reconstruction remains challenging, since ligaments represent heavily loaded tissues with high extracellular matrix (ECM) content, very low cell numbers, limited blood supply and as a consequence, restricted repair capacity. To circumvent limited auto graft availability and donor site morbidity, tissue engineered biological grafts could present benefit for ligament reconstruction in future. Valuable tissue engineered approaches can be developed based on small-scale in vitro models.

This mini review was prepared to draw attention to some experimental key problems and to illustrate approaches to establish tissue engineered ligament substitutes including appropriate scaffolds, functionalization of them, applicable cell sources, three-dimensional (3D) cell culturing and seeding strategies. It opens also the view on novel perspectives, which could move ligament tissue engineering forward such as utilization of various natural allogenic and xengenic ligament-derived ECMs and first attempts in ligament bioprinting.
\end{abstract}

Keywords: ligament, tendon, tissue engineering, ligament reconstruction
Volume 2 Issue 4 - 2017

\author{
Gundula Schulze Tanzil \\ Department of Anatomy, Paracelsus Medical University, \\ Germany
}

Correspondence: Gundula Schulze Tanzil, Institute of Anatomy, Paracelsus Medical University, Salzburg and Nuremberg, Prof. Ernst Nathan Str. I, 90419 Nuremberg, Germany, Tel +49(0)9। I-398-6772, Fax +49-(0)9II-398-6774,

Email gundula.schulze@pmu.ac.at, gundula.schulze-tanzil@klinikum-nuernberg.de

Received: May 28, 2017| Published: June 21, 2017

\section{Mini review}

Reconstruction of musculoskeletal tissues requires innovative strategies, which need to be developed by experimental research. As much as possible the application of tailored and optimized in vitro models based on human-derived cell sources should be expanded to substitute and scale down animal experiments and to collect sufficient information for the concerted selection of the most suitable and meaningful in vivo model. Three-dimensional (3D) culturing models are the only advisable systems for long-term culture of primary cells such as ligamentocytes. Complex direct or indirect 3D co-cultures allow studying the interaction between two or more cell types and present the basis for establishing miniaturized tissuelike in vitro models, ${ }^{1}$ including the ligament enthesis. Sophisticated bioreactors create an in vivo like micromilieu for $3 \mathrm{D}$ culturing and should be further developed to achieve much more inartificial culture conditions. In addition, some of them allow natural-like biomechanical training of the constructs supporting ligamento- or tenogenic cell differentiation. ${ }^{2-4}$

In regard to rebuilding mechano-sensitive and ECM-rich tissues such as bone, cartilage, tendon or ligament, biomaterial-based tissue engineering approaches are promising for tissue reconstruction: biomimetic biomaterials could transiently sustain biomechanic requirements of the natural tissue until the implanted cells have produced sufficient amounts of their own ECM. However, the biomaterial substituting a ligament should degrade very slowly, should not produce wear debris and has to maintain its stability for several months. To fulfil these and all other demands, developing biomaterialbased tissue engineering strategies for ligament reconstruction requires an intimate interaction between experts of material sciences, cell biology, engineers of biomechanics, orthopedists and several other disciplines to design, develop and approve suitable cell/biomaterial combinations. Unfortunately, until now no polymer system for ligament reconstruction entered the human clinic. ${ }^{5}$
Through this, various biomaterials have been selected and applied for ligament tissue engineering such as synthetic such as poly lactic acid (PLA) or polyurethan, as well as natural polymers such as collagen or silk and composites of both. ${ }^{6,7}$ Decellularized ECMs from allogenic or xenogenic donor-derived ligaments or tendons provide nearly natural tissue composition and biomechanics and therefore, present a promising approach for ligament tissue engineering. ${ }^{8,9}$ In addition, the ECMs promote differentiation of precursor cells. ${ }^{10,11}$ Scaffolds for ligament tissue engineering can be produced by various techniques such as braiding, embroidering, 3D printing, electrospinning, electro hydrodynamic jet printing and combinations of them..$^{6,10,12-15}$ Aligned, interwoven and multilayered composite structures most likely reflect the natural aligned ligament ultrastructure and biomechanics. ${ }^{10}$

Subsequently, an extensive biomaterial testing is necessary starting with the pure material to evaluate crucial parameters such as hydrophobicity/hydrophilicity, degradation, permeability, porosity, interconnectivity, surface texture and biomechanics. Later, in combination with a model cell line and then, with the respective ligamentogenic primary cell types, cellular parameters should be assessed such as cytocompatibility, cell adherence, distribution, morphology, cytoskeletal architecture, gene and protein expression profiles including established ligament and fibroblast markers such as scleraxis, tenomodulin and tenascin C. ${ }^{16}$ Unfortunately, many synthetic polymers such as PLA or natural polymers such as silk with promising mechanical properties allow only limited cell adherence and growth. ${ }^{6,7}$

Therefore, functionalization of synthetic polymers is demanded. ${ }^{12,13,17}$ Biomaterial functionalization should increase cell adherence, survival, spreading and migration, proliferation or differentiation and at least ligamentogenesis. It could also be applied to adapt biomechanical properties. ${ }^{18}$ For this purposes, various strategies have been undertaken to optimize polymer surfaces e.g., the supplementation of the material with natural ECM molecules such 
as biopolymers, which are characteristic for the respective tissue. In the case of tendons and ligaments collagen, prepared in various formulations (such as hydrogels, coatings, foams, sponges, films) led to promising results. ${ }^{6,19}$ Other functionalization strategies included the integration of motifs for fibronectin ${ }^{16}$ or RGD peptides into the scaffolds to improve integrin receptor-mediated cell adherence or the addition other functional groups. ${ }^{16,20}$ Several approaches used the slow release of stimulatory growth factors (GFs) attached to the scaffolds. ${ }^{14,17,21}$ All these modifications require many testing experiments under highly standardized conditions. The presence of stimulatory GFs or natural ligament ECM is especially advantageous if precursor cells are used to produce a tissue engineered neotendon or-ligament. ${ }^{11}$

Multipotent stromal cells (MSCs) are highly attractive for musculoskeletal tissue reconstruction due to their favorable properties such as high proliferative capacity, plasticity, differentiation potential, low immunogenicity, immunomodulatory capacity, trophic effects and higher availability. ${ }^{22}$ Harvesting MSCs presents lower donor site morbidity than harvesting primary tissue-specific resident cells such as ligamentocytes. ${ }^{16,23,24}$ Therefore, various types of stem cells including MSCs, induced pluripotent stem cells (iPSC), parthogenetic stem cells or embryonic stem cells have been explored for experimental tissue reconstruction and also for ligament reconstruction., ${ }^{3,25-28}$ However, embryonic stem cells can be excluded for ethical concerns and iPSC present still several risks and so far, do not reliably and fully reflect all properties of ESCs. ${ }^{25}$ Despite tenocytes or ligamentocytes are the predominant cell types in tendons and ligaments, arranged in longitudinal rows within the tissue, both tissues contain also some other cell types such as endothelial cells, synoviocytes, fibrochondrocytes and tendon stem cells. In this context, the enthesis zones of tendons and ligaments present a particular challenge for biological reconstruction. ${ }^{14,29}$

The question arises how to place the cells in an optimal position to allow functional neotissue formation as well as to guarantee the maintenance of a particular cell distribution and prospective tissue functionality. In ligaments and tendon, cells are aligned in rows. Bioprinting could be a strategy to place cells in a matrix in a defined position in rows and to combine them with vasculogenic cell types, since, vascularization of implants is necessary. A recent study described an ectopic in vivo incubation in the rabbit model to achieve vascularization before using the silk-collagen construct for anterior cruciate ligament (ACL) reconstruction. ${ }^{30}$ Strategies for vascularization can be explored in vitro using co-cultures. ${ }^{31-33}$ and further developed by bioprinting, which is an emerging field in tissue engineering. Attempts have already been undertaken to print a muscle-tendon unit. ${ }^{34}$ However, purely bioprinted scaffolds are usually hydrogel-based, because a defined rheology is required for the printing process and therefore, normally too weak to sustain natural ligament biomechanics. Bioprinting can be also used to print GFs into ligament scaffolds. ${ }^{35}$ Nevertheless, combining bioprinting technique with elastically extendable polymer scaffolds using a collagen-based bioink might be a versatile strategy in future to embed the cells or aggregates of them in a defined order into the scaffold structure to produce a composite ligament implant.

"Tissue on chip" based approaches allow miniatur-scaled experiments. ${ }^{36,37}$ Only a small amount of expensive biomaterials or GFs is necessary e.g. to test the effects of GFs or other mediators on tenogenesis by precursor cells or tenocytes. Following this approach, animal experiments can be reduced. ${ }^{38}$ Furthermore, testing of a large range of variations is possible under exactly adapted similar environmental conditions. However, except for an approach to establish fascicles in miniaturized size ${ }^{39}$ there are up to now no published models for tendon or ligament on chip available.

Numerous ligament injuries such as ACL ruptures happen yearly with increasing incidence. Ligament healing remains very time consuming and often incomplete or, in the case of ACL ruptures, mostly fails. Ligament healing can lead to scar formation with impaired functionality and the risk of re-rupturing. At many locations autografts represent the gold standard of ligament reconstruction, associated with limited availability and donor site morbidity. ${ }^{40}$ Therefore, biological tissue engineered constructs would be appreciated as a novel approach. So far no tissue engineering-based ligament reconstruction strategy is available for clinical application. Meanwhile, there exist novel perspectives since innovative biomimetic composite scaffolds and biocompatible natural ligament ECM based scaffolds have been developed. However, functionalization and automatized seeding strategies of scaffolds which possess appropriate biomechanic properties have to be further elaborated to facilitate functional neoligament formation and to acquire implants that fully fulfill clinical requirements. Whether proprioceptive capabilities of ligaments can be reestablished in implanted neo-ligaments in future presents also an unsolved question.

\section{Acknowledgements}

This small overview is associated with a project of the author supported by German Research Foundation (DFG, SCHU1979/14_1).

\section{Conflict of interest}

The author declares no conflict of interest.

\section{References}

1. Hendriks J, Riesle J, van Blitterswijk CA. Co-culture in cartilage tissue engineering. J Tissue Eng Regen Med. 2007;1(3):170-178.

2. Engebretson B, Mussett ZR, Sikavitsas VI. The effects of varying frequency and duration of mechanical stimulation on a tissue-engineered tendon construct. Connect Tissue Res. 2017;1:1-11.

3. Liu W, Yin L, Yan X, et al. Directing the differentiation of parthenogenetic stem cells into tenocytes for tissue-engineered tendon regeneration. Stem Cells Transl Med. 2017;6(1):196-208.

4. Mace J, Wheelton A, Khan WS, et al. The role of bioreactors in ligament and tendon tissue engineering. Curr Stem Cell Res Ther. 2016;11(1):3540 .

5. Sava OR, Sava DF, Radulescu M, et al. Trends in materials science for ligament reconstruction. Curr Stem Cell Res Ther. 2017;12(2):145-154.

6. Mozdzen LC, Vucetic A, Harley BA. Modifying the strength and strain concentration profile within collagen scaffolds using customizable arrays of poly-lactic acid fibers. J Mech Behav Biomed Mater. 2017;66:28-36.

7. Sharifi Aghdam M, Faridi Majidi R, Derakhshan MA, et al. Preparation of collagen/polyurethane/knitted silk as a composite scaffold for tendon tissue engineering. Proc Inst Mech Eng. 2017;H 1:954411917697751.

8. Gogele C, Schwarz S, Ondruschka B, et al. Decellularized iliotibial band recolonized with allogenic homotopic fibroblasts or bone marrow-derived mesenchymal stromal cells. Methods Mol Biol. 2017;doi:10.1007/7651 201730. 
9. Jones G, Herbert A, Berry H, et al. Decellularization and characterization of porcine superflexor tendon: a potential anterior cruciate ligament replacement. Tissue Eng Part A. 2017;23(3-4):124-134.

10. Rothrauff BB, Yang G, Tuan RS. Tissue-specific bioactivity of soluble tendon-derived and cartilage-derived extracellular matrices on adult mesenchymal stem cells. Stem Cell Res Ther. 2017;8(1):133.

11. Yang G, Rothrauff Benjamin B, Lin Hang, et al. Tendon derived extracellular matrix enhances transforming growth factor-beta3-induced tenogenic differentiation of human adipose-derived stem cells. Tissue Eng Part A. 2017;23(3-4):166-176.

12. Hahner J, Hoyer M, Hillig S, et al. Diffusion chamber system for testing of collagen-based cell migration barriers for separation of ligament enthesis zones in tissue-engineered ACL constructs. J Biomater Sci Polym Ed. 2015;26(16):1085-1099.

13. Hoyer M, Drechsel N, Meyer M, et al. Embroidered polymer-collagen hybrid scaffold variants for ligament tissue engineering. Mater Sci Eng C Mater Biol Appl. 2014;43:290-299.

14. Parry JA, Olthof MG, Shogren KL, et al. Three Dimension Printed Porous Poly (Propylene Fumarate) Scaffolds with Delayed rhBMP-2 Release for Anterior Cruciate Ligament Graft Fixation. Tissue Eng Part A. 2017;23(7-8):359-365.

15. Wu Y, Wong YS, Fuh JY. Degradation behaviors of geometric cues and mechanical properties in a 3D scaffold for tendon repair. J Biomed Mater Res A. 2016;105(4):1138-1149.

16. Rehmann MS, Luna JI, Maverakis E, et al. Tuning microenvironment modulus and biochemical composition promotes human mesenchymal stem cell tenogenic differentiation. $J$ Biomed Mater Res A. 2016;104(5):1162-1174.

17. Kuo CK, Marturano JE, Tuan RS. Novel strategies in tendon and ligament tissue engineering: Advanced biomaterials and regeneration motifs. Sports Med Arthrosc Rehabil Ther Technol. 2010;2:20.

18. Lipner J, Boyle JJ, Xia Y, et al. Toughening of fibrous scaffolds by mobile mineral deposits. Acta Biomater. 2017;58:492-501.

19. Hoyer M, Meier C, Breier A, et al. In vitro characterization of self-assembled anterior cruciate ligament cell spheroids for ligament tissue engineering. Histochem Cell Biol. 2015;143(3):289-300.

20. Marturano JE, Schiele NR, Schiller ZA, et al. Embryonically inspired scaffolds regulate tenogenically differentiating cells. J Biomech. 2016;49(14):3281-3288.

21. Pauly HM, Sathy BN, Olvera D, et al. Hierarchically structured electrospun scaffolds with chemically conjugated growth factor for ligament tissue engineering. Tissue Eng Part A. 2017;23(15-16):823-836.

22. Huang Z, Godkin O, Schulze Tanzil G. The challenge in using mesenchymal stromal cells for recellularization of decellularized cartilage. Stem Cell Rev. 2017;13(1):50-67.

23. Kang JW, Koo HC, Hwang SY, et al. Immunomodulatory effects of human amniotic membrane-derived mesenchymal stem cells. $J$ Vet Sci. 2012;13(1):23-31.
24. Ryan JM, Barry FP, Murphy JM, et al. Mesenchymal stem cells avoid allogeneic rejection. J Inflamm (Lond). 2005;2:8.

25. Bavin EP, Smith O, Baird AE, et al. Equine induced pluripotent stem cells have a reduced tendon differentiation capacity compared to embryonic stem cells. Front Vet Sci. 2015;2:55.

26. Dale TP, Mazher S, Webb WR, et al. Tenogenic differentiation of human embryonic stem cells. Tissue Eng Part A. 2017;24(5-6):361-368.

27. Ishiy FA, Fanganiello RD, Griesi Oliveira K, et al. Improvement of In Vitro osteogenic potential through differentiation of induced pluripotent stem cells from human exfoliated dental tissue towards mesenchymal-like stem cells. Stem Cells Int. 2015;2015: 249098.

28. Pillai DS, Dhinsa, BS, Khan W. Tissue engineering in achilles tendon reconstruction; the role of stem cells, growth factors and scaffolds. Curr Stem Cell Res Ther. 2017;12(6):506-512.

29. Liu H, Yang L, Zhang E, et al. Biomimetic tendon extracellular matrix composite gradient scaffold enhances ligament-to-bone junction reconstruction. Acta Biomater. 2017;56:129-140.

30. Ran J, Hu Y, Le H, et al. Ectopic tissue engineered ligament with silk collagen scaffold for ACL regeneration: A preliminary study. Acta Biomater. 2017;53:307-317.

31. Liu Y, Chan JK, Teoh SH. Review of vascularised bone tissue-engineering strategies with a focus on co-culture systems. J Tissue Eng Regen Med. 2015;9(2):85-105.

32. Lu Q, Simionescu A, Vyavahare N. Novel capillary channel fiber scaffolds for guided tissue engineering. Acta Biomater. 2005;1(6):607-614

33. Lu HH, Jiang J. Interface tissue engineering and the formulation of multiple-tissue systems. Adv Biochem Eng Biotechnol. 2006;102:91-111.

34. Merceron TK, Burt M, Seol YJ, et al. A 3D bioprinted complex structure for engineering the muscle-tendon unit. Biofabrication. 2015;7(3):035003

35. Ker ED, Nain AS, Weiss LE, et al. Bioprinting of growth factors onto aligned sub-micron fibrous scaffolds for simultaneous control of cell differentiation and alignment. Biomaterials. 2011;32(32):8097-8107.

36. Kallioniemi OP. Biochip technologies in cancer research. Ann Med. 2001;33(2):142-147.

37. Lueking A, Cahill DJ, Mullner S. Protein biochips: A new and versatile platform technology for molecular medicine. Drug Discov Today. 2005;10(11):789-794.

38. Baudoin R, Corlu A, Griscom L, et al. Trends in the development of microfluidic cell biochips for in vitro hepatotoxicity. Toxicol In Vitro. 2007;21(4):535-544.

39. Neal D, Sakar MS, Ong LL, et al. Formation of elongated fascicle-inspired 3D tissues consisting of high-density, aligned cells using sacrificial outer molding. Lab Chip. 2014;14(11):1907-1916.

40. Konrath JM, Saxby DJ, Killen BA, et al. Muscle contributions to medial tibiofemoral compartment contact loading following ACL reconstruction using semitendinosus and gracilis tendon grafts. PLoS One. 2017;12(4):e0176016 